\title{
Netherton Syndrome: Case Report and Review of the Literature
}

\author{
Maira E. Herz-Ruelas ${ }^{\text {a }}$ Sonia Chavez-Alvarez ${ }^{\text {a }}$ \\ Juana Irma Garza-Chapa ${ }^{a} \quad$ Jorge Ocampo-Candiani ${ }^{a}$ \\ Victor Andres Cab-Morales ${ }^{\mathrm{b}}$ David Emmanuel Kubelis-López ${ }^{\mathrm{a}}$
}

aDepartment of Dermatology, University Hospital "Dr. José E. González," Universidad Autónoma de Nuevo León, Monterrey, Mexico; 'baculty of Medicine and University Hospital “Dr. José E. González," Universidad Autónoma de Nuevo León, Monterrey, Mexico

\section{Keywords}

Netherton syndrome $\cdot$ Trichorrhexis invaginata $\cdot$ Ichthyosis linearis circumflexa

\begin{abstract}
Netherton syndrome (NS) is a rare genodermatosis with an autosomal recessive pattern of inheritance caused by pathogenic variants in the SPINK5 gene. It is characterized by a triad consisting of atopic diathesis, ichthyosis linearis circumflexa, and hair shaft abnormalities. Ichthyosis linearis circumflexa can be confused with atopic dermatitis leading to a delayed diagnosis. Furthermore, difficulty in making the differential diagnosis with other atopiform, erythrodermic, and ichthyosiform entities that exhibit hair shaft abnormalities represent a challenge. Trichoscopy is an accessible and noninvasive auxiliary diagnostic tool in these cases; the hair shaft abnormalities found in NS are bamboo, golf tee, and matchstick hairs. Identification of a pathogenic variant in the SPINK5 gene through genetic testing is necessary to confirm the diagnosis. Multiple treatment options are available including topical therapy with emollients, corticosteroids, calcineurin inhibitors, antiseptics, and narrowband UVB phototherapy. Systemic treatments comprehend intravenous im-
\end{abstract}

munoglobulins, and advances in the understanding of the pathophysiology of NS have led to more directed therapies with biologics such as infliximab, ixekizumab, secukinumab, ustekinumab, and dupilumab. Treatments currently under investigation include inhibitors of kallikrein 5, cathelicidins, drugs activating the transcription factor nuclear factor erythroid-derived 2-like 2, and gene therapy using autologous keratinocytes induced with a lentiviral vector encoding SPINK5.

(c) 2021 S. Karger AG, Basel

\section{Introduction}

Netherton syndrome (NS) is a rare and potentially mortal autosomal recessive genodermatosis with an incidence of 1 case per 200,000 newborns [1,2]. The characteristic findings of NS include icthyosis linearis circumflexa (ILC) described by Comel in 1949, consisting of pruritic polycyclic erythematous patches with a double-edged circinate or serpiginous scale. Another feature is the bamboo hair (trichorrhexis invaginata) that comprises an invagination of the hair shaft giving a "ball in cup" appearance, pathognomonic hair abnormality of NS, and de- 
Fig. 1. a Erythematous and serpiginous plaques with characteristic double-edged marginal scale. b Eczematous lesions involving the eyelids and oral commissures. Lack of external portion of the eyebrows.
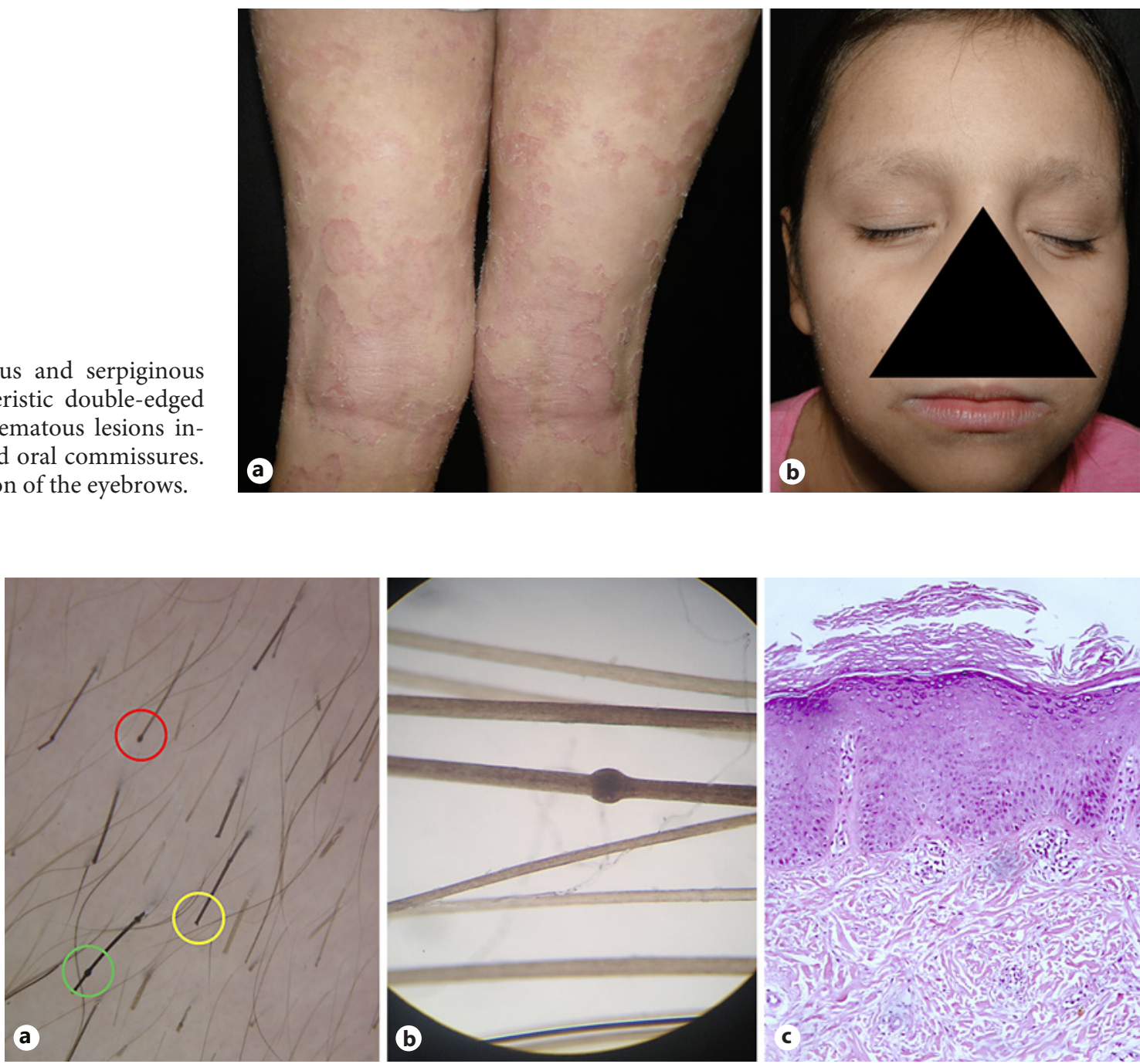

Fig. 2. a Dermoscopy of eyebrows. Bamboo hairs (green), golf tee hairs (yellow) and matchsticks hairs (red). b Trichorrhexis invaginata, confirmed with optical microscope from a sample of the occipital region. c Histopathology with hematoxylin and eosin stain at $\times 20$ magnification showing hyperkeratosis, acanthosis, focal hypergranulosis, and superficial perivascular lymphocytic infiltrate.

scribed by Netherton in 1958. Last, the former 2 findings and atopic diathesis conform the well-known triad first described by Wilkinson in 1964 [1-3].

\section{Case Report}

A 7-year-old Hispanic girl presented to our clinic referring a recalcitrant intensely pruritic dermatosis, along with sparse hair. The mother referred that she began at birth with scalp lesions and subsequent development of disseminated intermittent eczematous plaques. Physical examination revealed erythematous and squamous plaques with double-edged desquamation showing polycy- clic and serpiginous borders (Fig. 1a). Also, a lack of the external portion of the eyebrows was noted as well as short and brittle hair predominantly in the occipital region (Fig. 1b). Within her personal history, she had recurrent respiratory tract infections that required in-hospital management and a family history of a sister deceased with acute lymphoblastic leukemia.

Trichoscopy of the eyebrows showed bamboo hairs, golf tee, and matchstick hairs (Fig. 2a); trichorrhexis invaginata was observed predominantly in the occipital region (Fig. 2b). Skin biopsy exhibited hyperkeratosis, acanthosis, focal hypergranulosis, and superficial perivascular lymphocytic infiltrate (shown in Fig. 2c). Laboratory findings were remarkable for peripheral eosinophilia and increased immunoglobulin E levels. The patient was given topical mild-potency corticosteroids, oral antihistamines, and 
emollients. Narrowband UVB phototherapy was considered but since the patient lived in the suburbs, it was difficult for her to adhere to treatment. She was referred for genetic counseling and to the department of allergy and immunology for further evaluation.

\section{Discussion}

\section{Pathogenesis}

Around 80 different pathogenic variants causing loss of function in SPINK5 (serine protease inhibitor Kazaltype 5) gene have been identified in NS [4,5]. This gene is localized on chromosome $5 \mathrm{q} 32$ and encodes a serine protease inhibitor called lymphoepithelial Kazal-type-related inhibitor (LEKTI), expressed in the thymus and epithelia [2]. Deficiency in LEKTI causes an unrestricted activity of the epidermal serine proteases kallikrein 5 (KLK 5) and kallikrein 7 (KLK 7) [6]. KLK 5 activity degrades desmoglein 1, leading to an increased desquamation of the stratum corneum resulting in an altered skin barrier function. This could explain the recurrent bacterial infections and may also facilitate allergen penetration contributing to the atopic diathesis found in NS $[2,6,7]$. Another consequence of KLK 5 hyperactivity is the activation of nuclear factor kappa B (NF-kB) through the signaling of protease-activated receptor 2 (PAR2), culminating in an increased expression of tumor necrosis factor $a$ (TNF- $\alpha$ ), interleukin-8, and thymic stromal lymphopoietin (TSLP) [2, 6]. TSLP overexpression induces T cells toward a $\mathrm{T}$ helper type 2 phenotype and increases levels of immunoglobulin $\mathrm{E}$ which has a role in the atopic manifestations of NS $[2,7]$. In recent studies, a prevailing T helper type 17 profile has been found in NS that has also been explored therapeutically [8].

\section{Clinical Manifestations}

The clinical manifestations of NS may begin at birth or during the first postpartum weeks. Skin findings are diverse with diffuse xerosis, erythema and lichenification at flexural sites, urticaria, angioedema, and congenital ichthyosiform erythroderma. The more severe cases may present collodion membrane at birth. Eventually the pathognomonic skin manifestation ILC develops but may wax and wane $[1,4,6]$. Clinically, the hair is short, weak, scant, and dull; eyebrows are sparse, especially the lateral portions. Evaluation under the microscope or with trichoscopy reveals the pathognomonic sign of trichorrhexis invaginata. Also, golf tee hairs can be observed as a result of the rupture of the hair shaft at the level of invagination leaving the cup-shaped portion of the shaft. An- other hair shaft abnormality that can be encountered is the matchstick sign consisting of a broken hair shaft with a bulging end. The aforementioned hair shaft defects may be found in the scalp, eyebrows, and eyelashes, but some authors report that trichorrhexis invaginata and golf tee are more frequently found in the eyebrows compared to the scalp [3]. Pili torti, trichorrhexis nodosa, and helical hairs are other hair shaft abnormalities reported in NS [1].

Other manifestations of NS include chronic noninfectious diarrhea, hypereosinophilia, allergy to cow's milk, intellectual disability, neurologic deficits, short stature, asthma, allergic rhinitis, hypogammaglobulinemia, and hypergammaglobulinemia $[1,4]$. Complications include hypernatremic dehydration, altered thermoregulation, failure to thrive, infections, and sepsis [2, 4]. Follow-up of patients with NS must include regular dermatological examinations because of the increased risk of skin cancer reported [9].

\section{Diagnosis}

Diagnosis of NS is suggested by the clinical findings but can be difficult due to multiple reasons: ILC is not always present, hair examined under light microscopy may lack the hair shaft abnormalities as it affects only $20-50 \%$ of hairs, and atopic manifestations may mask the clinical picture [1]. Histology is nonspecific but a negative LEKTI immunostaining may suggest the diagnosis, though few cases are LEKTI positive, so genetic testing is necessary to confirm the diagnosis $[2,4]$. Differential diagnoses include autosomal recessive congenital ichthyosis, Omenn syndrome, generalized seborrheic dermatitis, erythrodermic psoriasis and staphylococcal scalded skin syndrome $[1,10]$.

\section{Treatment}

In a recent guideline for the management of congenital ichthyoses, the first line of treatment is topical therapy. Emollients are recommended at least 2 times a day and preferably after bathing; in areas of skin inflammation, erosions, or flexural sites, emollients without urea are preferred. To prevent recurrent infections, antiseptics (dilution of 5 parts of chlorhexidine in 1,000-10,000, octenidine $0.1 \%$, polyhexanide $0.1 \%$, dilution of 1 part of potassium permanganate in 10,000 , or diluted bleach $0.005 \%$ solution) are recommended 2-3 times a week [10]. The selection and duration of topical treatments in NS is important, considering the increased absorption secondary to the skin barrier defect. Topical corticosteroids are recommended for short periods due to the risk of Cushing syndrome and skin atrophy. Topical calcineurin inhibitors are also recommended for limited time and 
in localized areas during flares [9]. Other therapies include narrowband UVB phototherapy, which may be efficacious but is recommended for short term due to the risk of skin cancer in patients with NS [9]. Another systemic treatment are intravenous immunoglobulins which are safe and effective but, because of the limited evidence available, are not recommended for long-term use [9]. The advances in the understanding of the pathophysiology of NS have led to more directed therapies with biologics. For example, because of the increased expression of TNF- $\alpha, 2$ patients were treated with infliximab, reporting improvement of skin lesions, as well as longer and stronger hair. However, the usage of TNF- $\alpha$ inhibitors is not recommended, considering that patients under these therapies have an increased risk of infections and skin cancers, risks already present in this population $[2,7,9]$. In a case report, a decrease in allergic skin symptoms was observed with omalizumab [9]. Another reported therapeutic strategy is the blockage of the Thelper type 17 axis with ixekizumab, an anti-interleukin-17 A/F antibody; clinical improvement was described in 3 patients during the induction phase and was partially sustained in the maintenance phase [8]. Secukinumab, an anti-interleukin $17 \mathrm{~A}$ antibody, has been used as well in 4 patients with severe NS, reporting a reduction by $44-88 \%$ in the Ichthyosis Area and Severity Index total score after 3 months of therapy, along with a reduction by $40-76 \%$ of Dermatology Life Quality Index and by $27-62 \%$ in the $5-\mathrm{D}$ itch scale. Sustained improvement was observed at 6-month follow-up [11]. Ustekinumab blocks the p40 subunit of interleukin-12 and interleukin-23. A case report observed substantial improvement with no relapse after 1 year of 3-monthly treatment [5]. Last, suppression of interleukin- 4 and interleukin-13 by dupilumab greatly improves pruritus and scaling, but partial improvement in erythema has been observed [12]. Further investigation is necessary on all these new targeted therapies to better determine which patients may benefit from these treatments.

Multiple investigations of novel targets for the treatment of NS are currently in progress. Because of the known increased activity of KLK 5 in NS, several specific inhibitors of KLK 5 are under development [13]. Cathelicidins, a group of antimicrobial peptide precursors, seem to be implicated in the skin inflammation in NS in a murine model; the authors suggested that drugs aiming to reduce the expression of cathelicidins combined with inhibitors of KLK 5 could be a promising therapy [13]. Another murine model studied the activation of the transcription factor nuclear factor erythroid-derived 2-like 2 (NRF2) which induced the overexpression of secretory leukocyte protease inhibitor (SLPI), an inhibitor of KLK 7 and elastase 2 leading to an improvement in the epidermal barrier defect, proposing that a drug activating NRF2 could be an effective treatment [14]. A recent phase 1 trial confirmed the safety and feasibility of gene therapy using autologous keratinocytes induced with a lentiviral vector encoding SPINK5, although still in initial phases is a potential treatment option [15].

\section{Conclusion}

NS is a rare genodermatosis. Its diagnosis may be delayed because atopic features and skin manifestations may be confused with atopic dermatitis [2]. Furthermore, difficulty in making the differential diagnosis with other atopiform, erythrodermic, and ichthyosiform entities that exhibit hair shaft abnormalities represent a challenge. Trichoscopy is an accessible and noninvasive auxiliary diagnostic tool in these cases, allowing a prompt diagnosis and thus adequate treatment [3].

\section{Statement of Ethics}

Written informed consent was obtained from the patient's mother for publication of this case report and any accompanying images.

\section{Conflict of Interest Statement}

The authors have no conflicts of interest to declare.

\section{Funding Sources}

No funding was received for the preparation of this manuscript.

\section{Author Contributions}

Maira E. Herz-Ruelas, MD, PhD, and Victor Andres Cab-Morales: idea conception, bibliographic review, writing of the original draft, writing, editing, and revision of the final manuscript. Sonia Chavez-Alvarez, MD: detailed trichoscopic findings and editing and revision of the final manuscript. Juana Irma Garza-Chapa, MD: description of the histopathological findings and editing and revision of the final manuscript. Jorge Ocampo-Candiani, MD, PhD: editing and revision of final the manuscript. David Emmanuel Kubelis-López, MD: bibliographic review, writing, editing, and revision of the final manuscript. 


\section{References}

1 Sun JD, Linden KG. Netherton syndrome: a case report and review of the literature. Int J Dermatol. 2006 Jun;45(6):693-7.

2 Roda Â, Mendonça-Sanches M, Travassos AR, Soares-de-Almeida L, Metze D. Infliximab therapy for Netherton syndrome: a case report. JAAD Case Rep. 2017 Nov;3(6):5502.

3 Meltem Akkurt Z, Tuncel T, Ayhan E, Uçmak $\mathrm{D}$, Uluca U, Uçak H. Rapid and easy diagnosis of Netherton syndrome with dermoscopy. J Cutan Med Surg. 2014 Jul-Aug;18(4):280-2.

4 Bellon N, Hadj-Rabia S, Moulin F, Lambe C, Lezmi G, Charbit-Henrion F, et al. The challenging management of a series of 43 infants with Netherton syndrome: unexpected complications and novel mutations. Br J Dermatol. 2020 Jun.

5 Volc S, Maier L, Gritsch A, Aichelburg MC, Volc-Platzer B. Successful treatment of Netherton syndrome with ustekinumab in a 15-year-old girl. Br J Dermatol. 2020 Jul; 183(1):165-7.

6 Small AM, Cordoro KM. Netherton syndrome mimicking pustular psoriasis: clinical implications and response to intravenous immunoglobulin. Pediatr Dermatol. 2016 May; 33(3):e222-3.
7 Fontao L, Laffitte E, Briot A, Kaya G, RouxLombard P, Fraitag S, et al. Infliximab infusions for Netherton syndrome: sustained clinical improvement correlates with a reduction of thymic stromal lymphopoietin levels in the skin. J Invest Dermatol. 2011 Sep;131(9): 1947-50.

8 Barbieux C, Bonnet des Claustres M, de la Brassinne M, Bricteux G, Bagot M, Bourrat E, et al. Duality of Netherton syndrome manifestations and response to ixekizumab. J Am Acad Dermatol. 2020 Jul.

9 Mazereeuw-Hautier J, Hernández-Martín A, O'Toole EA, Bygum A, Amaro C, Aldwin M, et al. Management of congenital ichthyoses: European guidelines of care, part two. Br J Dermatol. 2019 Mar;180(3):484-95.

10 Mazereeuw-Hautier J, Vahlquist A, Traupe H, Bygum A, Amaro C, Aldwin M, et al. Management of congenital ichthyoses: European guidelines of care, part one. Br J Dermatol. 2019 Feb;180(2):272-81.
11 Luchsinger I, Knöpfel N, Theiler M, Bonnet des Claustres M, Barbieux C, Schwieger-Briel A, et al. Secukinumab therapy for Netherton syndrome. JAMA Dermatol. 2020 Aug; 156(8):907-11.

12 Süßmuth K, Traupe H, Loser K, Ständer S, Kessel C, Wittkowski H, et al. Response to dupilumab in two children with Netherton syndrome: improvement of pruritus and scaling. J Eur Acad Dermatol Venereol. 2021 Feb; 35(2):e152-5.

13 Zingkou E, Pampalakis G, Sotiropoulou G. Cathelicidin represents a new target for manipulation of skin inflammation in Netherton syndrome. Biochim Biophys Acta Mol Basis Dis. 2020 10;1866(10):165831.

14 Muzumdar S, Koch M, Hiebert H, Bapst A, Gravina A, Bloch W, et al. Genetic activation of $\mathrm{Nrf} 2$ reduces cutaneous symptoms in a murine model of Netherton syndrome. Dis Model Mech. 2020 06;13(5):dmm.042648.

15 Di WL, Lwin SM, Petrova A, Bernadis C, Syed F, Farzaneh F, et al. Generation and clinical application of gene-modified autologous epidermal sheets in Netherton syndrome: lessons learned from a phase 1 trial. Hum Gene Ther. 2019 09;30(9):1067-78. 